\title{
Un análisis de contenido dual. Propuesta metodológica para el estudio de Internet como fuente
}

\author{
MYRIAM REDONDO \\ Universidad Carlos III de Madrid \\ myriam.redondo@gmail.com
}

Recibido: 10.05 .2007

Aceptado: 29.05 .2007

\section{INTRODUCCIÓN}

Pese a la rica e interesante bibliografía que existe sobre el periodismo en Red en nuestro país, a Internet se le han dedicado escasos acercamientos como fuente de información. El espacio digital suele abordarse como «nuevo medio» para el que trabajan «nuevos periodistas» (los periodistas digitales). No obstante, en realidad sólo un determinado porcentaje de reporteros trabaja para publicaciones digitales, mientras que todos los profesionales de la información son ciberperiodistas en cuanto que no sólo acuden al espacio digital quienes publican en él, sino también quienes pretenden extraer datos del mismo. De hecho, es previsible que en el futuro la mayoría de los periodistas se acerquen a la Red con esta segunda intención aún más que con la primera, y que además lo hagan con tanta naturalidad como hoy contestan al teléfono.

En particular, el uso de la Red como fuente de información en las secciones de Internacional de los medios se presenta como uno de los campos de observación más atractivos dentro del ámbito de la Comunicación. Al fin y al cabo, ofrece un caso práctico de cómo el engolado debate sobre la globalización tecnológica toma cuerpo en los contenidos palpables y diarios que se ofrecen finalmente a los lectores. Precisamente, entre las capacidades 'revolucionarias' que los autores más optimistas han adjudicado a Internet está la de permitir el acceso a contenidos globales, volviendo doméstico eso que antes se denominaba «el extranjero».

Este ángulo de observación (mirar a la Red como la fuente que es para los profesionales de cualquier tipo de medio, analógico o digital) no sustituye ni anula las aportaciones académicas de distinta vertiente, centradas en Internet 
como medio (los periódicos en su traslación a la Red, los blogs, los nuevos periodistas multimedia, etc.). Antes bien, puede servir para complementarlas, y por tanto parece provechoso.

En 2005 se planteó la realización de un estudio sobre la materia y, en concreto, sobre el modo en que la Web puede resultar útil a los periodistas dedicados a la información internacional (ya sea desde una corresponsalía o como redactores de mesa en la sede central de su medio). El resultado fue la tesis doctoral Internet como fuente de información en el Periodismo Internacional, en la que se detectan qué rutinas de contacto con las fuentes informativas despliegan los corresponsales españoles en Bruselas y se analiza si la Red podría ayudar a mejorar esas rutinas (Redondo, 2005b).

Las rutinas periodísticas pueden describirse como pautas de comportamiento consolidadas en la profesión que se asimilan por costumbre, se ejecutan de forma mecánica y están presentes en todo el proceso de producción informativa (Túñez, 1999: 148). Se derivan de los llamados valores noticiosos, criterios de selección que se ponen en marcha en las redacciones determinando qué es noticia y qué no; provocan, a su vez, lo que se conoce en su expresión general como «estereotipos», imágenes aceptadas previamente y con carácter inmutable que no se corresponden del todo con la realidad externa.

Valores noticiosos, rutinas y estereotipos han sido objeto de numerosos estudios académicos que se engloban en las llamadas news value research o newsmaking research. Con algunas variantes, estas dilatadas líneas de investigación tratan de definir qué condicionantes influyen a los periodistas al hacer su trabajo, es decir, qué factores impiden que la realidad contada por ellos se corresponda más fielmente con el mundo que habitamos. Una lista no excluyente de autores que han realizado aportaciones teóricas importantes al campo de la «fabricación de la noticia» debería incluir obligatoriamente a: Walter Lipmman (uno de los primeros en referirse a los «estereotipos» periodísticos); Gaye Tuchman (con su definición de las «estrategias rituales» que siguen los profesionales para no arriesgarse a disentir); P. Golding y P. Elliot (quienes propusieron la vigencia de «reglas prácticas» entre los redactores); E. Goffman (desvelando los frames o marcos que determinan el acercamiento a la información); Herbert Gans (al incidir en la influencia de las fuentes más habituales sobre la información); y S. Iyengar y D. R. Kinder, que defienden el priming o predisposición del periodista hacia unos asuntos $\mathrm{u}$ otros.

Pese a que Internet se ha hecho un hueco evidente en las rutinas periodísticas, no existen estudios monográficos sobre la interacción corresponsales-Red, sino tan sólo declaraciones dispersas y testimonios escritos por los propios periodistas internacionales. En eso radica parte del interés de este estudio, que además supone una apuesta metodológica significativa por dos motivos: a) aporta datos cuantitativos — «cuánta» Red utilizan los corresponsales-en un ámbito donde predominan las apreciaciones cualitativas y polarizadas (Internet acabará con la figura del corresponsal / el poder de Internet es un cuento); y $b$ ) aborda las 
rutinas periodísticas mediante una técnica poco aplicada a ellas, el análisis de contenido.

Es preciso aclarar que este artículo - como la tesis que lo precede- se apoya en las propuestas teóricas precedentes (reseñadas en la Bibliografía) y no trata de avanzar en esta dimensión. Antes bien, se centra en exponer su aplicación práctica a un marco informativo concreto (los contenidos internacionales), bajo un factor influyente específico (Internet) e incidiendo, más que en los resultados, en la metodología que puede seguirse para obtenerlos.

Incluso mostrando las complejidades y limitaciones encontradas en su diseño, el estudio puede servir para allanar el camino de otros trabajos sobre la Red como fuente, o al menos alentar la expansión de este tipo de investigaciones. Con ese propósito siempre abierto a mejoras y perfeccionamientos se presenta la investigación en Empiria.

\section{UN ANÁLISIS DE CONTENIDO COMPARATIVO}

¿Cómo saber de qué manera puede influir la Red como proveedora de información en la sección de Internacional de los medios (en realidad, también en cualquier otra sección interesada en lo que sucede en el extranjero)? Antes de nada, conviene definir qué Red configura el objeto de estudio, y aclarar de antemano que Internet no se aborda como fuente en sí misma sino como plataforma de acceso a los verdaderos informantes ${ }^{1}$. En esta propuesta, la Red es un:

«Sistema de fuentes de información que presenta sucesos o permite el acceso a individuos, entidades, documentos o archivos (entendidos en la más amplia variedad de formatos digitales posibles) que aportan pistas o materia prima para la elaboración de una noticia, que permiten la verificación y contraste de una noticia o que aportan noticias ya elaboradas a partir de las cuales es posible realizar un mero proceso de lectura - en el caso de los usuarioso un proceso de reelaboración informativa —en el caso de los periodistas-》 (Redondo, 2005b: 200).

Desde el prisma teórico del newsmaking, marca la tradición que el estudio de las rutinas se base en alguna de las siguientes metodologías: encuestas, entrevistas u observación participante (abierta o encubierta). Apoyándonos en esa variedad de propuestas, y en la riqueza de los datos que nos han ofrecido, sugerimos aquí que la digitalización de los contenidos y su presentación en Red abren nuevas vías complementarias - y no sustitutivas - para estudiar el proceso de fabricación de la noticia.

Como apuntó hace ya tiempo Adam Smith, la informatización está sacando a la luz numerosos mecanismos periodísticos antes ocultos. Sobre todo, se hacen

${ }^{1}$ No obstante, expresiones del tipo «Internet como fuente», se repiten a lo largo de este artículo con el objetivo de agilizar el texto.

EMPIRIA. Revista de Metodología de Ciencias Sociales. N. ${ }^{\circ}$ 13, enero-junio, 2007, pp. 35-58.

ISSN: $1139-5737$ 
más claras tanto la operación de documentación del reportero como los lugares de los que extrae algunos de sus datos (Smith, 1983: 250). En este proceso de reflote, las fuentes quedan expuestas más que nunca.

Partimos de la creencia de que encuestas, entrevistas y observación participante han demostrado su validez en el estudio de las rutinas de los periodistas. Si ahora buscamos transformar sus preguntas más habituales en variables de un análisis de contenido es para comprobar cuáles son los resultados mediante otro tipo de disección. La objetividad absoluta en una investigación es prácticamente imposible; sin embargo, distintas perspectivas pueden facilitar una comprensión más completa del objeto de estudio.

¿Qué aspectos del análisis resultarían beneficiados empleando un análisis de contenido, en lugar de las otras metodologías reseñadas? Responder a esta pregunta obliga a destacar los puntos débiles de encuestas, entrevistas y observación, sin que ello signifique — se recuerda una vez más - que tales técnicas deban ser desestimadas.

Reconocen algunos autores que en las encuestas y entrevistas quienes contestan tienden a contar lo que creen que han hecho o lo que les gustaría hacer más que lo que hicieron en realidad, mostrando sus cualidades y ocultando sus defectos (Piñuel y Gaitán, 1995: 439; Berger, 1991: 41). Por otra parte, el recurso a determinadas fórmulas lingüísticas para que los entrevistados o encuestados elijan una opción sin cansarse con excesivos detalles («poco, mucho o nada», por ejemplo), conduce a la relatividad e impide representar fielmente los acontecimientos y las situaciones (Gunter, 2002: 228).

A la hora de estudiar el funcionamiento de las corresponsalías, existe una larga tradición de uso de ambas técnicas -especialmente encuestas-. Pero como observa John Crothers Pollock, la investigación se hace más cuesta arriba cuando se entra en detalles sobre las fuentes empleadas por los corresponsales. Entonces, según el autor, conseguir un listado sutil de categorías es difícil y puede acarrear dificultades metodológicas. La tarea de los periodistas en este campo, dice Pollock, es demasiado compleja, y la pregunta « ¿dónde consigues tu información?» suele ser respondida con desgana (Pollock, 1981: 141).

La técnica de la observación participante -la más utilizada en los estudios de rutinas periodísticas, como constata Mauro Wolf- tampoco se libra de algunas dificultades. A veces conlleva una relajación de las normas establecidas para sistematizar o lleva al temido going native, un proceso por el que el investigador se contagia de la mentalidad de las personas analizadas (Wolf, 1987: 211-212).

Ninguna de estas dificultades - aunque puedan encontrarse otras, ya que ningún método es infalible - está presente en un análisis de contenido; por eso, uno prolongado en el tiempo y diseñado para detectar las rutinas periodísticas a través de sus pistas digitales parece una herramienta que suma, y no resta, a las investigaciones precedentes. Como sostiene Gaye Tuchman, el empleo del análisis de contenido para estudiar procesos de newsmaking no debe entenderse despectivamente como una «casta separada», sino como una vía complementaria de enorme utilidad. Y ello es así porque los textos noticiosos clara- 
mente atestiguan el proceso periodístico que les ha dado lugar, constituyéndose en «objetos necesarios de análisis» (Tuchman, 2002: 81-82).

Con la realización de un análisis de contenido que tiene en cuenta unidades digitales estamos además apoyando una de las tareas investigadoras de crecimiento más obligado. Si bien la mayoría de los estudios internacionales que se emprenden sobre la Comunicación están basados en esta técnica (Tsang, K. J., Tsai, Y. y Liu, S. K., 1988), su aplicación todavía es tentativa en terreno digital, donde los contenidos son animales cambiantes de difícil categorización ${ }^{2}$.

Todo estudio se realiza con un fin, y en este sentido conviene mostrar la hipótesis que sirvió de hilo conductor a la investigación de origen (Redondo, 2005b) para no perder el sentido de esta incursión metodológica. En dicha obra, el punto de partida hipotético era la apreciación optimista de que «Internet se configura como un sistema de fuentes beneficioso para el periodista internacional, con capacidad para transformar sus rutinas de producción y de relación con los actores informantes». Esta creencia implicaba salir en busca de evidencias relativas a tres circunstancias:

- La Red proporciona un porcentaje lo suficientemente elevado de noticias como para ser tenida en cuenta entre los puntos diarios de búsqueda de información.

- La Red facilita algunas de las rutinas periodísticas más repetitivas.

- La Red corrige posibles tendencias indeseables que se dan entre los profesionales, como el recurso excesivo a las declaraciones realizadas por fuentes de información de carácter privilegiado y elitista.

El objetivo del presente artículo no es descubrir si se confirman o no estas tres hipótesis, puesto que tal cometido ya ha sido realizado (Redondo, 2005a; Redondo, 2007), sino describir el camino metodológico que se siguió para su resolución, materia en la que se especializa Empiria. No obstante, y como línea de llegada, al final de este artículo se ofrece un esquema de las conclusiones alcanzadas, así como una justificación de su validez.

Para confirmar una hipótesis, y más si quien aborda el análisis es un investigador individual, el marco geográfico ha de ser reducido. La elección en este caso ha sido Bruselas, una ciudad que hace posible el estudio por tres motivos: es un foco informativo concreto e identificable, garantiza el acceso de los periodistas a Internet y es terreno activo de fuentes que comunican acontecimientos por la vía digital. En definitiva, se trata de un escenario que, al menos a priori, puede proporcionar suficientes pruebas analizables como para que la investigación no quede invalidada de antemano. Pese a que ésta es la opción propuesta aquí, otros supuestos serían también válidos. Así, es plausible el análisis

${ }^{2}$ El 84\% de las investigaciones publicadas en las principales revistas científicas internacionales de comunicación se basan en la metodología del análisis de contenido, según el estudio efectuado por K. J. Tsang, Y. Tsai y S. K. Liu. 
de la Red como fuente periodística para informar sobre un país o zona geográfica (Estados Unidos, Irak, Hispanoamérica, etc.) o para realizar pesquisas temáticas (periodismo económico, taurino, etc.).

¿Puede un análisis de contenido captar las relaciones que entablan fuentes y periodistas? Se sostiene que a través de la comparación sí: con un análisis de contenido paralelo de los mensajes de las fuentes por un lado y los mensajes de los medios por otro, no parecen existir motivos para dudar de que la relación deducida entre ambos sea fiable. En este sentido, se han emprendido ya algunas investigaciones similares que profundizan en la influencia de los gabinetes de comunicación, o de sus notas de prensa, sobre los contenidos publicados por los medios. Una de ellas es la realizada por Vladimir de Semir, Cristina Ribas y Gemma Revuelta ya en 1998, que reflejaba la influencia de los comunicados emitidos por las grandes revistas científicas en los artículos que los medios deciden publicar.

Con esos comunicados expuestos en la Web, el análisis de contenido dual es aún más posible. La secuencia de acción es la siguiente: se toman las informaciones publicadas en varios medios españoles sobre noticias relativas al marco geográfico o temático elegido (en este caso, Bruselas) y se busca, para cada una de ellas, un archivo digital previo que pudiera haber servido como fuente en Internet. La comparación entre ambos planos es la que ofrece los resultados del análisis.

Siendo obligada la comparación (digital-papel), ninguno de los modelos de análisis de contenido existentes cuando se emprendió la investigación parecía conveniente para evaluar dos esferas tan distintas sin resultar forzado en su aplicación a alguna de las dos. No obstante, frente a la opción de construir desde cero un procedimiento propio se consideró más conveniente partir de un modelo ya testado sobre el terreno de la comunicación internacional. Las noticias internacionales y el nuevo orden en la información mundial, de los investigadores apoyados por la UNESCO Robert L. Stevenson y Donald L. Shaw (Stevenson y Shaw, 1985), sirvió como camino a seguir.

Este libro recoge parte de las conclusiones de una investigación emprendida por la UNESCO en 1980 sobre el funcionamiento de los flujos internacionales de noticias. Con dicha investigación, la institución internacional pretendía comprobar si esos flujos perjudicaban sistemáticamente al Tercer Mundo y ofrecían estereotipos negativos sobre el mismo, tal y como denunciaban algunos impulsores del conocido Informe McBride. Stevenson y Shaw, miembros del equipo internacional designado por la UNESCO para realizar el análisis, se adelantaron a la publicación de los resultados generales con la divulgación de los datos obtenidos desde Estados Unidos.

Pese a la controversia originada por este adelanto unilateral, lo cierto es que el informe final publicado posteriormente por la UNESCO (Sreberny-Mohammady, Nordenstreng, Stevenson et al., 1985) vino a sostener las conclusiones de los investigadores estadounidenses, aunque con algún matiz. En líneas generales, Stevenson y Shaw apuntaban que la norma informativa verdaderamente vigente 
en aquel momento era que la propia región geográfica concentrase mayor atención que cualquier otra parte del mundo. Pero más que esta revelación, lo interesante para el caso que nos ocupa está en la preparación del trabajo. En su proceso de diseño los propios autores reconocieron numerosas dificultades para acordar lo que deberían ser las líneas generales de un proyecto tan ambicioso (llegaron a calificarlo de «imposible»). Finalmente, fueron limados los aspectos más localistas para llegar al consenso, estableciéndose categorías con sentido en todos los países del estudio. Este pulido pragmático convierte la investigación en una de las más avanzadas metodológicamente ${ }^{3}$.

La metodología empleada por la UNESCO es la que se ha aplicado en esta propuesta, aunque con notables modificaciones. Los cambios se centran en dos aspectos: la introducción del concepto de «unidades de registro digitales» y la ampliación de los parámetros relativos a las fuentes, el ámbito que dejó más insatisfechos a los investigadores de la UNESCO (Sreberny-Mohammady, Nordenstreng, Stevenson et al., 1985: 53).

Con relación a lo primero, el de «unidades de registro digitales» es más que un nuevo concepto, y supone dar una segunda dimensión con entidad propia al estudio primigenio, donde Internet estaba lógicamente ausente de toda consideración (la investigación se produce en los años 80). A partir de este desarrollo dual, y dada la necesidad de comparación continua (entre la versión digital y la versión papel), era obligada la introducción de toda una serie de variables que aclarasen la relación entre uno y otro plano (como son las de «clave informativa» $\mathrm{y}$ «clave ambiental», que se explican más adelante).

En cuanto a las fuentes, fue necesario acudir a la tesis doctoral que José Manuel Rivas Troitiño elaboró en los años 90 sobre ETA (Rivas Troitiño, 1992). De este trabajo - ejemplo de que un análisis de contenido puede reflejar de manera fehaciente las rutinas periodísticas - se tomaron los conceptos de «Regla» y «Estrategia de información», con los que esta investigación está completamente en deuda.

El plan previsto se simplifica si se opta por la inspección de medios de comunicación escritos (y no audiovisuales), pues un cotejo «texto a texto» siempre resulta más revelador. No hay que olvidar que el texto sigue siendo el lenguaje que predomina en Internet, y tampoco que, según todos los indicios, son los periódicos los que parecen seguir fijando la agenda mediática, mientras las televisiones repican sobre los temas que los rotativos han lanzado. Así pues, la opción de esta investigación fue la de centrarse en periódicos y no en canales de televisión. Sin embargo, no hay nada que impida realizar, con algunas modificaciones categóricas, un análisis comparado de medios audiovisuales-fuentes digitales.

${ }^{3}$ El llamado «Foreign images study» de la UNESCO es, en palabras de Thomas Haniszch, uno de los que «mejor ha superado la prueba del tiempo» dentro de la comunicación internacional (Hanisz, 2006). Prueba de ello es que su metodología ha inspirado numerosos estudios posteriores de referencia. El coordinado por R. L. Stevenson y A. Sreberny (Sreberny y Stevenson, 1997) es el ejemplo más claro que puede ofrecerse, tanto por el número de países implicados (38) como por el de unidades de información analizadas (más de 48.000). 
En cuanto al número de diarios observados y al período de vigilancia, lo ideal es examinarlos a todos y durante el mayor tiempo posible. Sin embargo, si es obligatorio optar entre ambos extremos (o muchos medios o mucho tiempo) es preferible la segunda opción, ya que sólo el largo plazo permite una detección fidedigna de las rutinas.

En el ejemplo que se está explicando, los medios elegidos fueron El Mundo y El País, por su alta representatividad ideológica. En cuanto al período de análisis, se amplió la horquilla esbozada en Las noticias..., donde se examina un trimestre entero basándose en una muestra de dos semanas, una natural y otra compuesta (Stevenson y Shaw, 1985: 37-54) ${ }^{4}$. En nuestra investigación, los meses elegidos son los que transcurrieron entre julio del año 2003 y junio del año 2004, ambos incluidos, lo que supone cuatro trimestres. Extrayendo dos semanas de cada uno de ellos se obtuvo un total de ocho semanas (56 días) como representación de un año completo ${ }^{5}$. Como semanas naturales se escogieron al azar (aunque esquivando conscientemente los meses de verano) las segundas semanas completas del tercer y quinto mes de cada semestre: septiembre y noviembre de 2003 y marzo y mayo de 2004, respectivamente.

Como hemos dicho, en el estudio se habla de «unidades de registro papel» y «unidades de registro digitales». Las primeras se entienden como «texto informativo publicado en prensa, encabezado por un titular y con presencia de firma o localización». Para evitar repeticiones cacofónicas, reciben nombres como «registro papel», «pieza informativa», «artículo»o «noticia». Estas dos últimas menciones, sin embargo, no guardan relación con la distinción formal entre géneros informativos (noticia, crónica, reportaje, entrevista, columna de opinión o editorial), que no fue tenida en cuenta en la investigación.

Las unidades de texto encabezadas por un titular pero carentes de datos geográficos y de autoría no pudieron ser analizadas. Los despieces que sólo presentaban titular se analizaron como parte de la crónica mayor a la que pertenecían, y aquellos que presentaban — además de titular- firma o lugar, se consideraron como noticia separada.

En segundo lugar se analizan las unidades de registro digitales, de muy diverso tipo y que podrían describirse como «documentos o archivos de texto, audio o vídeo emitidos por las fuentes en Internet y que comunican -o presentan a personajes que comunican - un hecho novedoso propicio a ser convertido en noticia por los medios». Los registros digitales, también denominados a lo largo del estudio «archivos-fuente», «registros-fuente» o «registros digitales», cubren un amplio espectro que va desde archivos de vídeo en formato MPEG hasta las tradicionales notas de prensa, ahora enviadas por e-mail o presentadas

${ }^{4}$ La semana natural es elegida al azar, mientras para formar la semana compuesta se toma un día de cada quince: señálese el primer lunes del trimestre estudiado y, a partir de él, cuéntense quince días para determinar el siguiente martes, el siguiente miércoles, el siguiente jueves, etc.

${ }_{5}$ Es necesario realizar una puntualización. La UNESCO consideró semanas que iban sólo de lunes a sábado, pero aquí se incluyen los domingos, lo que llevó a la modificación de algunos detalles metodológicos (Redondo, 2005b: 265). 
en la Red. En total, la muestra del análisis que se maneja como ejemplo estuvo comprendida por 293 unidades de registro papel y 125 unidades digitales.

Tras la lectura de cada unidad de registro en papel se procede a localizar su precedente digital. En este caso, la búsqueda no debe reducirse a un abanico cerrado de sitios web sino que ha de estar abierta a toda la Red. Conocer de antemano el terreno digital con el que va a encontrarse el investigador es aconsejable, sobre todo porque lo más visible (y con lo que uno está tentado a contentarse) son las llamadas Salas de Prensa Digitales (SPD). Pueden definirse como «espacios web situados dentro del sitio general de una entidad o individuo y cuyo objetivo específico es el de ofrecer información y servicios a la prensa».

Las SPD son espacios que suelen estar guiados por profesionales de la información y corresponden por tanto a fuentes ávidas con una estrategia de comunicación bien definida. Internet es mucho más que eso, y parte de su fuerza radica en su espontaneidad desordenada y amateur. Por eso debe comprobarse si sitios menores o alternativos, más vinculados al llamado periodismo ciudadano que a actores oficiales o empresariales, pueden resultar también fuentes útiles.

En el caso de Bruselas, se conocía de antemano que la oferta digital era amplia y variada, comprendiendo desde numerosas páginas web oficiales (como el elefantiásico Sitio Europa) hasta direcciones alternativas mucho menos conocidas pero también valiosas ${ }^{6}$. Sin embargo, una de las decepciones principales del análisis se produjo al comprobar cómo, pese al enorme abanico disponible, siete SPD oficiales terminaban revelándose como fundamentales y aglutinando buena parte de la información útil para la Prensa: las dependientes de la Comisión Europea, el Parlamento Europeo y el Consejo de ministros de la Unión Europea (UE), accesibles desde el Sitio Europa (Imagen 1); la del Comité de Representación Permanente de España en Bruselas (REPER); las dos puestas en marcha por las presidencias semestrales italiana e irlandesa de la UE; y la de la OTAN $^{7}$.

\section{VARIABLES Y CATEGORÍAS}

El listado completo de las variables contempladas (se consignaron hasta 20) queda reflejado en los formularios con los que se rellenó la base de datos del análisis (la Imagen 2 es la plantilla que se usó para los archivos en papel; la Imagen 3, para las unidades digitales). A continuación se especifican los detalles de los rasgos que otorgan particularidad a este estudio o introducen un cambio notable (total o parcial) con respecto a las investigaciones principales en las que se apoya, especialmente las de la UNESCO y Rivas Troitiño. Como el lector ob-

\footnotetext{
${ }^{6}$ En el Sitio Europa (http://www.europa.eu.int) pueden consultarse todas las páginas web oficiales de la Unión, pero también hay vida fuera de él.

7 REPER: http://www.es-ue.org; presidencia irlandesa de la UE: http://www.eu2004.ie/; presidencia italiana de la UE: http://www.ueitalia2003.it (ya no puede consultarse); OTAN: http:// www.nato.int
}

EMPIRIA. Revista de Metodología de Ciencias Sociales. N. ${ }^{\circ}$ 13, enero-junio, 2007, pp. 35-58.

ISSN: $1139-5737$ 


\section{Press Room}

\begin{tabular}{|c|c|}
\hline UROPA > E & Headlines | Latest Press Rele \\
\hline Drocos: & Press Packs \\
\hline Database & $\begin{array}{l}\text { Iraq . European Council, } 17-18 \text { June } 2004 \text {. The Lisbon Strategy - Sustainabl } \\
\text { CAPIDoha. Middle East Constitution for Europe Enlargement MTO-Cancu }\end{array}$ \\
\hline Last 7 days & America Summit = EU-US Summit, 25-26/06/2004 \\
\hline
\end{tabular}

\section{Midday express}

Subscribe

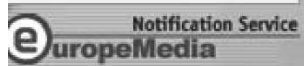

News in photos

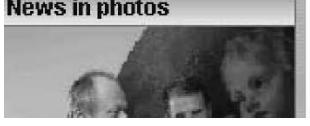

\section{Diteritinos}

$10 / 09 / 04$

\section{Commission clears the acquisition of Srnstar by HP de}

Commission authorises EDP acquisition of sole control over Hidrocanti fict

"Seize opportunity of EU rural development monev", EU Farm Commis

Imagen 1. Portada de la Sala de prensa digital de la Comisión Europea.

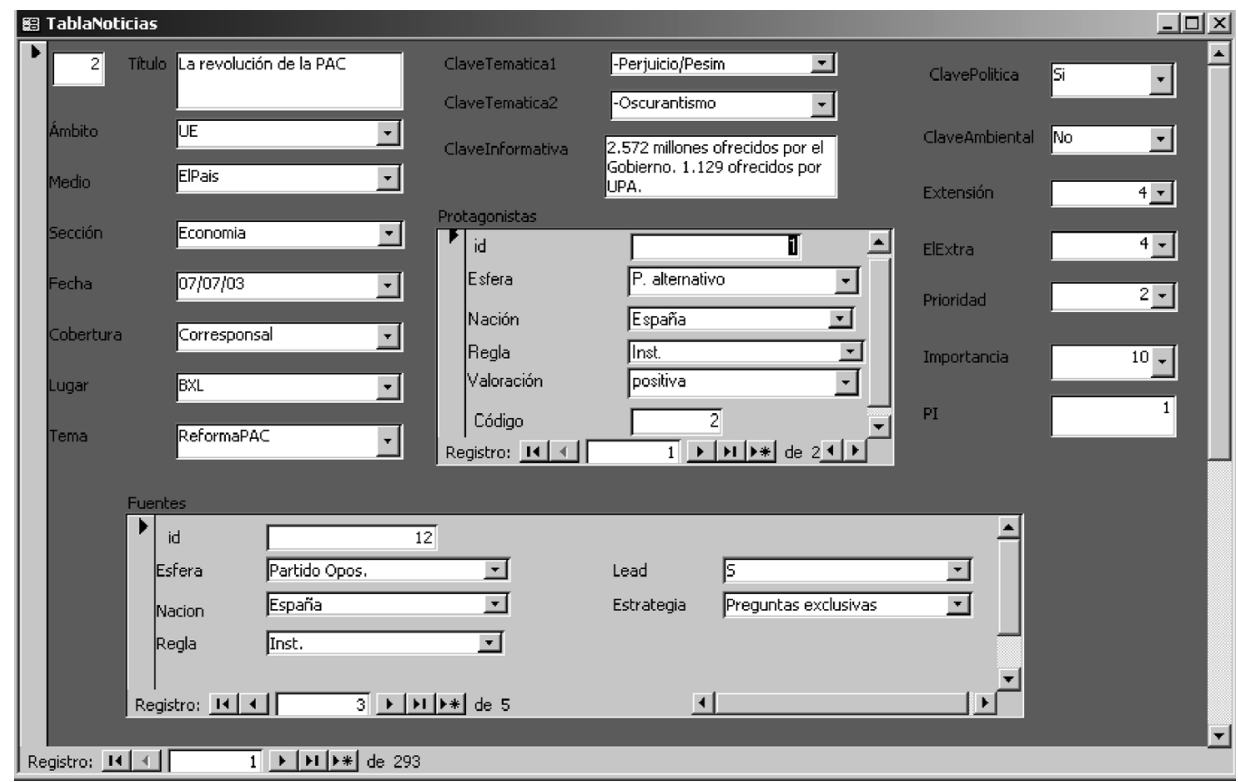

Imagen 2. Formulario de las unidades de registro papel.

servará, cada una de las variables que van siendo reseñadas es aplicable tanto a las unidades de registro papel como a las unidades de registro digitales, lo que permite el cotejo final. 


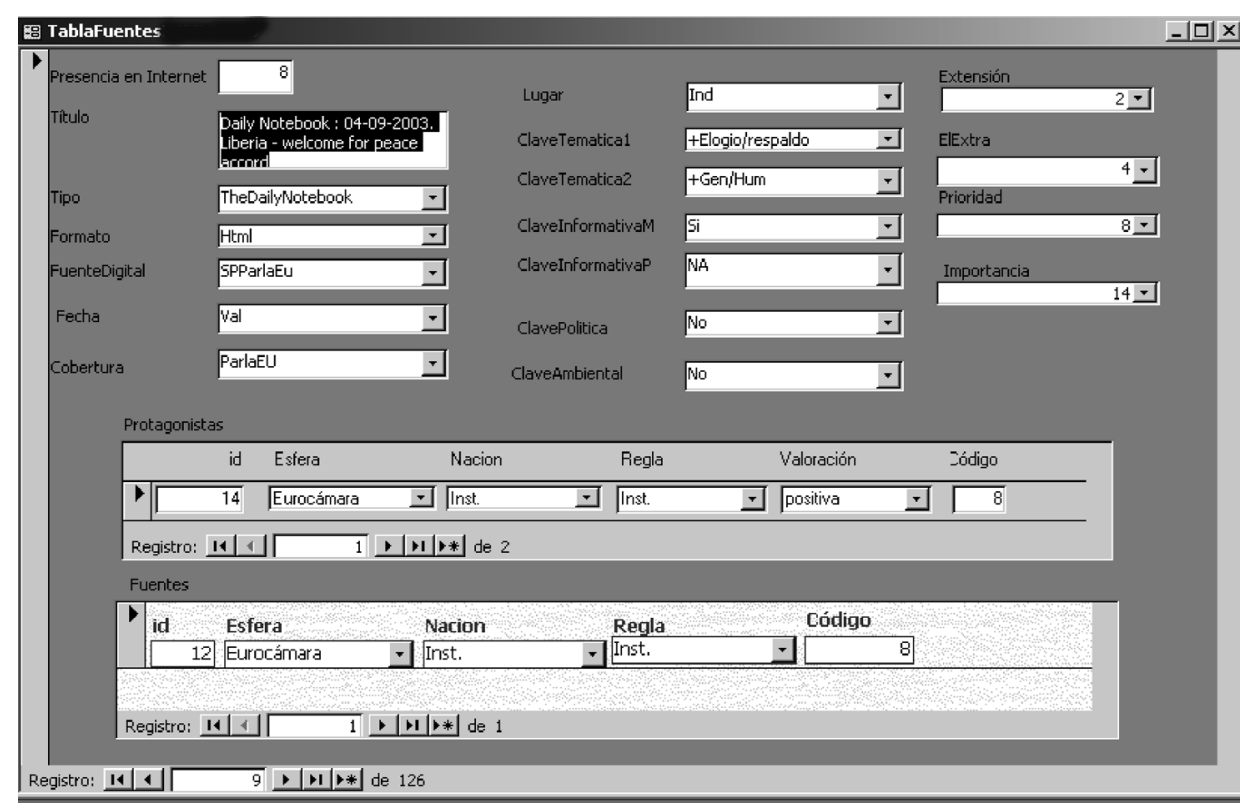

Imagen 3. Formulario de las unidades de registro digitales.

Antes de ofrecer el listado, se recuerda dónde está la prioridad. Son variables fundamentales las relativas a tres puntos directamente relacionados con las hipótesis de partida: a) hasta qué punto los datos que facilitan las fuentes se encuentran en la Red antes de que los periodistas elaboren la noticia (variable «presencia en Internet»); b) qué rutinas y tipos de relación entre fuentes y periodistas pueden deducirse del análisis y pueden simplificarse en Red («estrategias»); y c) qué tipo de fuentes originan el grueso de la información que publican los periodistas (variable «fuentes» y todas sus subvariables derivadas).

Estas son las más importantes:

1. Medio/Fuente digital. Indica el rotativo del que procede la unidad papel analizada y contempla dos categorías: El País y El Mundo. En el caso de los archivos online, esta variable se denominó «fuente digital», ya que la palabra «medio» resultaba limitadora y parecía no hacer justicia a la variedad de publicaciones en Red. La «fuente digital» es el sitio web (de la modalidad que sea, desde una SP hasta un blog) en el que la persona o institución hace público el acontecimiento. Una clasificación plausible de «fuentes digitales» sería la siguiente: a) SP empresariales o institucionales; b) agencias y medios de comunicación tradicionales; c) revistas exclusivamente digitales (elaborados por profesionales); d) blogs; e) medios colaborativos (elaborados por ciudadanos); f) foros o chats; y g) otros. 
2. Fecha. Sirve para detectar posibles diferencias en el tratamiento periodístico efectuado durante un lapso de tiempo y otro (por ejemplo, entre un tramo de normalidad política y otro de convocatoria electoral).

En los registros papel las categorías de la variable «Fecha» corresponden a cada uno de los días de la muestra, pero en los registros digitales el interés era otro: comprobar si estaban disponibles con tiempo suficiente para que el periodista pudiera utilizarlos como fuente. Por ello se consignaron tres categorías: a) válida: el registro digital estaba presente en la Red el día anterior a la publicación de la noticia; b) inválida: el registro apareció en la Red el mismo día de su publicación o posteriormente; c) indeterminada: no se especifica en qué momento el archivo adoptó presencia digital, y por tanto su validez no puede considerarse garantizada.

Con relación a la fecha, cada investigador debe determinar el nivel de exigencia deseado. Si los medios que se analizan son audiovisuales, el umbral de validez desciende (basta con que el registro digital esté presente en la Red minutos antes de la emisión de la noticia).

3. Caracteres. Junto a «Elemento extra», «Prioridad» e «Importancia» constituye el conjunto de variables formales que confirma la categoría otorgada por el periódico a la pieza informativa. Tomando como referencia una noticia a toda página de El País, que puede contener unos 7.000 caracteres sin espacios, se consideraron en esta variable tres categorías: a) noticia pequeña, de 0 a 2.000 caracteres sin espacios: la pieza recibe 2 puntos; b) noticia mediana, de 2.001 a 4.000: recibe 4 puntos; c) noticia grande, más de 4.000: recibe 6 puntos ${ }^{8}$.

En cuanto a los archivos de Internet, los de tipo textual fueron clasificados en pequeños, medianos y grandes siguiendo la pauta aplicada a las noticias. A los archivos en formato de audio o vídeo se les aplicó una medida en minutos: pequeños (de 0 a 10 minutos), medianos (de 11 a 20) y grandes (de 21 en adelante). Cada categoría recibe la misma puntuación que en el caso de las noticias.

4. Elemento extra. Dentro de esta variable, si la noticia se acompaña de fotografías o gráficos, se codifica la categoría «presente», que recibe 4 puntos; en caso contrario, «ausente», que dispone sólo de dos.

Para las unidades de registro digitales se consignará la categoría «presente» (4 puntos) cuando el archivo ostente los siguientes rasgos: a) si es un documento en formato HTML, Word o PDF, que incluya fotografías, gráficos o vídeos (en su defecto, que presente enlaces o hipervínculos a otras páginas web, a documentos con más información o a archivos de audio o vídeo); b) si es un archivo de audio o vídeo, siempre.

La categoría «ausente» se consigna en los casos contrarios.

${ }^{8}$ La especificación de los caracteres totales por cada noticia no se considera necesaria dado que el presente análisis de contenido se basa en el número de informaciones y no en el espacio que éstas ocupan. Según el estudio de Stevenson y Shaw, apenas existen diferencias entre ambas modalidades de análisis, mientras el primero resulta más sencillo (Stevenson y Shaw, 1985: 51-53). 
5. Prioridad. Denota la visibilidad de la noticia dentro del conjunto del periódico. Categorías: a) portada/destacado: si aparecen en primera página tanto el titular como el primer párrafo de la noticia, el registro recibe 8 puntos; b) portada: sólo el titular de la noticia aparece en primera página, recibiendo la pieza informativa 6 puntos; c) destacado: noticias que no aparecen en primera página pero sí están destacadas en su sección, recibiendo 4 puntos; d) nulo: noticia que no aparece en primera página ni ha sido destacada en su sección. Recibe 2 puntos.

En el caso de los archivos digitales, la variable Prioridad estuvo entre las más difíciles de regular. Y ello porque los sitios web no respetan las características de los medios tradicionales de información. Pueden no aportar páginas numeradas, ni portada fija diaria, u ofrecer pasado el tiempo las noticias en un almacén desde donde no se conoce si aparecieron destacadas o no.

Durante el análisis de la información procedente de Bruselas, el criterio que mejor permitió determinar la importancia de los archivos digitales fue el número de idiomas en el que aparecían replicados. Así, se estimó que una nota de prensa mostraba mayor jerarquía informativa cuando era traducida al mayor número posible de lenguas de la Unión.

Con este criterio, las categorías resultantes fueron las siguientes: a) español y otros, 8 puntos (equivalente a portada/destacado); b) español, 6 puntos (equivalente a portada); c) más de dos idiomas europeos, excluido el español, 4 puntos (destacado); d) dos idiomas europeos o menos de dos, excluido el español, 2 puntos (nulo) ${ }^{9}$. Evidentemente, esta clasificación debe ser modificada en otro lugar donde el multilingüismo no sea santo y seña del sistema informativo que se está analizando.

6. Importancia. Con ella se cierra el conjunto de variables formales; recoge los parámetros de las tres anteriores y resume el nivel jerárquico de la noticia o del registro digital. Categorías: a) extremadamente alta, 18 puntos; b) muy alta, 16 puntos; c) alta, 14 puntos; d) normal, 12 puntos; e) baja, 10 puntos; f) muy baja, 8 puntos; g) extremadamente baja, 6 puntos. Con la variable «Importancia» se puede deducir si las noticias que germinan en línea son verdaderamente significativas o tan sólo sirven de relleno al medio.

7. Tema. Es el tipo de asunto al que la noticia se refiere principalmente y que es compartido por la noticia y por su archivo digital. Los temas fueron analizados en dos niveles; el primero, siguiendo parcialmente la clasificación ideada por el equipo estadounidense de la UNESCO, divide las noticias por categorías generales; el segundo, novedad aquí, las distingue dentro de esa primera separación y especifica qué casos o «hilos informativos» concretos fueron objeto de atención en el periodo analizado.

${ }^{9}$ Cuando la nota aparecía en español se entendía que, si bien podía no existir una excesiva importancia objetiva, sí la tenía para los corresponsales españoles.

EMPIRIA. Revista de Metodología de Ciencias Sociales. N. ${ }^{\circ}$ 13, enero-junio, 2007, pp. 35-58.

ISSN: $1139-5737$ 
Los hilos temáticos serán siempre específicos en cada investigación que se emprenda (por ejemplo, uno muy habitual durante el estudio fue el juicio al pederasta belga Marc Dutroux ${ }^{10}$ ). Se detectan cuando un mismo asunto ocupa a los medios más de un día. Al consignar los temas y los hilos, el análisis permite precisar aún más que por secciones qué rasgos comunes tienen los asuntos periodísticos que brotan en Internet.

En cuanto a las categorías generales, deudoras de la UNESCO aunque no exactamente iguales, son las siguientes: a) actividad política interior; b) actividad política y diplomática exterior; c) conflictos internos (incluye elecciones o cambios de Gobierno); d) ejército y defensa, relativo a conflictos armados o amenazas de conflictos armados; e) terrorismo, sobre políticas, conflictos o negociaciones con grupos terroristas o amenazas de grupos terroristas; f) materias económicas, incluyendo todo lo relativo a comercio, industria, relaciones laborales, concentraciones de empresas... etc.; g) desastres naturales; h) sociedad, en noticias que abordan la salud, la vivienda, la religión..., etc.; i) crimen, policía y asuntos judiciales; j) ciencia y tecnología; k) cultura, artes, espectáculos; l) deportes; m) ecología; y por último n) interés humano, que aglutina noticias sobre acontecimientos sorprendentes o conmovedores, así como referidas a perfiles personales.

8. Clave temática principal. Refleja el ánimo con el que la fuente hace público el contenido o con el que el periodista narra la noticia que tiene entre manos. Permite dilucidar qué tono predomina en las informaciones que llegan al público y, sobre todo, qué tono predomina en los comunicados válidos que pueden encontrarse en línea. ¿Tienen carácter crítico o, por el contrario, invitan a una cierta inacción?

En esta variable, las categorías se dividen en tres grandes grupos (positivas, negativas y grupo neutro), de modo que cada opción de signo + cuenta con una antagonista. Son las siguientes (entre paréntesis, la negativa): a) apertura/transparencia (oscurantismo); b) beneficio/optimismo (perjuicio/pesimismo); c) claridad/sencillez (confusión/complicación); d) coordinación/unidad interna (descoordinación/división interna); e) coordinación/unidad externa (descoordinación/división externa), con referencia a las acciones emprendidas dentro de un grupo de países; f) cumplimiento (o incumplimiento), se refiere a aquellas ocasiones en las que se actúa según lo acordado o bajo el respeto a la ley; g) elogio/respaldo (crítica/reclamación), donde se presenta a un actor avalando a otro o reconociendo su actuación como meritoria; h) entusiasmo/firmeza (languidez/dubitación); i) generosidad/solidaridad (cicatería/insolidaridad); j) logro/eficacia/acierto (retroceso, ineficacia, error), donde se llega a una situación buscada y esperada desde hace tiempo; k) seguridad/fortaleza (peligro/debilidad); 1) neutra, no puede detectarse interpretación alguna.

${ }^{10}$ Marc Dutroux fue detenido en 1996 tras secuestrar, torturar, violar y asesinar a varias menores. Su captura, tardía y plagada de errores, mostró una enorme incompetencia policial y política.

EMPIRIA. Revista de Metodología de Ciencias Sociales. N. ${ }^{\circ}$ 13, enero-junio, 2007, pp. 35-58. ISSN: 1139-5737 
9. Clave informativa. Novedad en el análisis. Es el dato o declaración necesaria y suficiente de la noticia, normalmente contenido en su titular y entradilla, a partir del cual se desarrolla la narración y sin el cual ésta no hubiera sido posible.

En las unidades papel, se trata una variable de categorización abierta, ya que puede haber tantas claves informativas como noticias. El germen de una noticia es particular y específico. Si se repite, sólo puede hacerlo en otro medio y sobre el mismo asunto.

En las unidades de registro digitales se contemplaron dos claves informativas posibles: la de El Mundo y la de El País, dado que un mismo registro digital puede contener el dato que vertebra la noticia en el rotativo de Pedro J. Ramírez pero no la publicada por el diario del Grupo PRISA, o al revés.

Un mismo archivo digital puede aportar dos claves informativas (tanto $E l$ Mundo como El País la convirtieron en noticia), una (lo hizo un medio pero otro no) o ninguna (los medios convirtieron el tema tratado por el registro digital en noticia pero centrándola en un aspecto no contenido en él). En los dos primeros casos, la categoría consignada es «presente»; en el tercero, «ausente». Para que un registro digital sea considerado útil, es fundamental que incluya la clave informativa del artículo papel con el que se relaciona. De otra forma, se tratará de un archivo referido a idéntico tema pero que no habrá servido de nada al periodista.

10. Clave ambiental. Una noticia con clave ambiental es aquella en la que, añadiéndose a la clave informativa, cobra una gran importancia el modo en que se comportaron o el aspecto que presentaban ciertos protagonistas de la acción. Dicho de otra manera, en las noticias con clave ambiental se hace necesaria la observación directa del periodista, que puede describir matices formales que de otro modo quedarían ocultos para el lector. Un ejemplo: puede ser noticia el hecho de que George Bush visite un país extranjero y, en rueda de prensa, dé a sus anfitriones las gracias por la acogida; puede ser noticia de claro tinte ambiental que la comunicación no verbal revele en ese momento una hostilidad de fondo sólo parcialmente disimulada por las grandilocuentes declaraciones.

Las categorías de esta variable, tanto para el plano digital como para el papel, son «presente» $\mathrm{y}$ «ausente». Si el registro digital no tiene clave ambiental pero la noticia a la que hace referencia tampoco, ese registro sigue siendo útil. Sin embargo, si la noticia presenta rasgos ambientales y el registro precedente no, su eficacia como fuente es nula, dado que hurta ciertos aspectos imprescindibles de lo que aconteció.

11. Fuente. La fuente es la persona o material de la que el periodista obtiene su información. Puede ser, por lo tanto, un individuo, un documento o un grupo/entidad que informa de modo colectivo.

El carácter neurálgico de esta variable llevó a escudriñarla a través de cinco subvariables: esfera, regla, nacionalidad, entradilla y estrategia. 
11.1. Esfera: Es el ámbito de actividad del que surge el documento o en el que desempeña su labor la persona que está facilitando la información. Con la subvariable Esfera es posible averiguar hasta qué punto las fuentes a las que se recurre son verdaderamente plurales o sólo variantes de una misma élite compuesta por agentes oficiales y privilegiados.

Las esferas a las que se recurrió en Internet como... son muy detalladas y específicas de Bruselas (Consejo, Comisión, Parlamento..., etc.). Pero existen categorías más genéricas que pueden aplicarse a cualquier otro análisis. Por ejemplo: instituciones internacionales, gobierno, partidos políticos, cuerpo judicial, cuerpo diplomático, realeza, Ejército y Fuerzas y Cuerpos de la Seguridad del Estado (FCSE), Iglesia, empresa/patronal, agencias/Medios de Comunicación de Masas (MCM), medios nacidos en el entorno digital, celebridades, intelectuales/expertos, grupos terroristas/criminales, fuentes alternativas, fuentes indeterminadas (aquellas sobre las que ni siquiera se nos dice en qué ámbito desempeñan su actividad) y otras.

En este listado de categorías, podrían considerarse fuentes oficiales las siete primeras mencionadas; fuentes no oficiales serían las restantes. En cuanto a «fuentes privilegiadas» serían todas excepto los medios digitales, los grupos terroristas o criminales y las fuentes alternativas. La categoría «fuentes no privilegiadas» queda para las demás opciones ${ }^{11}$.

Conviene precisar la noción de fuentes alternativas, que suponen el mayor conjunto dentro de las no privilegiadas. Son aquéllas ajenas a las esferas donde se toman las grandes decisiones políticas, económicas o militares. Se trata de actores que informan, al menos en teoría, de manera independiente, sin vínculos con quienes ostentan el poder. Pueden citarse, entre otros ejemplos, las fundaciones, centros de análisis, ONGs, plataformas vecinales, publicaciones limitadas al entorno digital o la propia ciudadanía cuando se expresa ${ }^{12}$. En el presente estudio, la categoría alternativa sólo podría adjudicarse a las que se han denominado fuentes alternativas, a los medios crecidos en el entorno digital y a los grupos terroristas y criminales.

11.2. Regla. Concreta si la fuente es de tipo genérico (institución) o se trata de un cargo personal. Con ello revela el nivel jerárquico de los actores informantes más recurrentes y refuerza las pistas obtenidas mediante la variable anterior sobre la preferencia por fuentes privilegiadas o no privilegiadas.

${ }^{11}$ Es preciso incidir en el hecho de que no siempre las fuentes privilegiadas son oficiales. Greenpeace, por ejemplo, es una fuente no oficial pero privilegiada, puesto que su capacidad económica y tradición informativa dota de enorme impacto a sus mensajes.

${ }^{12}$ Es cierto que algunas ONG y centros de análisis pueden ser consideradas actores tan privilegiados como lo sería un cargo político electo. También se ha señalado que muchas pueden funcionar como grupos cercanos a los gobiernos (Grant, 1989: 16-19). Sin embargo, a la hora de clasificarlas se les otorgó el beneficio de la duda. Se trata de organizaciones que, a priori, trabajan con objetivos ajenos al de mantener a los poderosos, por lo que pueden llevar el título de «alternativas».

EMPIRIA. Revista de Metodología de Ciencias Sociales. N. ${ }^{\circ}$ 13, enero-junio, 2007, pp. 35-58. ISSN: 1139-5737 
También aquí las categorías fueron abordadas con un sesgo específico de Bruselas, pero puede pensarse en otras más generales: institución, jefe de Estado, presidente de Gobierno, presidente de organización internacional, ministro, juez, político, diplomático, periodista, experto/intelectual, militar, policía, deportista, celebridad, ciudadano e indeterminada. Excepto la primera categoría, todas las demás serían personales.

11.3. Entradilla o lead. Refleja si la fuente aparece o no en la entradilla o primer párrafo de la noticia. Si es así, son consideradas de mayor importancia, puesto que orientan la línea principal del artículo. Dentro de la subvariable entradilla sólo existen dos categorías posibles: presente y ausente.

11.4. Nacionalidad. Refleja el origen geográfico de la fuente informante y sirve para determinar qué discursos territoriales predominan en la información internacional. Las categorías son las siguientes: a) institucional, cuando la fuente no representa sólo a un país sino a una entidad supranacional o internacional; b) Estados Unidos; c) Alemania; d) Reino Unido; e) Francia; f) Italia; g) España; h) Japón; i) China; j) India; k) Israel; l) Irak; m) Rusia; n) Cuba; ñ) otras naciones europeas; o) otras naciones asiáticas; $p$ ) otras naciones latinoamericanas; q) naciones africanas; r) naciones de Oceanía; y s) indefinida, si la nacionalidad permanece velada.

11.5. Estrategia. Esta subvariable, que no se midió en el caso de los archivos digitales, refleja el modo en que el periodista se relaciona con la fuente y obtiene su información. Por su importancia, sus categorías se definen pormenorizadamente:

a) Acto público. Remite a las declaraciones efectuadas en inauguraciones, plenos o comparecencias públicas.

b) Documento. Comunicados, estudios, informes o notas de prensa emitidas por las fuentes.

c) Rueda de prensa. Encuentro específicamente planteado por la fuente para transmitir información a los periodistas.

d) Pasillos. Información facilitada en encuentros con la Prensa que no habían sido organizados de antemano, al menos de manera explícita. Según se desprende del estudio, en realidad se trata de declaraciones imprevistas sólo aparentemente y que no pierden intencionalidad.

e) Filtración. Informes orales o escritos entregados por la fuente al periodista y relativos a una materia que no estaba destinada a hacerse pública. Dentro de «informes orales» se incluyen, por ejemplo, las explicaciones «anónimas» que reciben los periodistas sobre las anécdotas que ocurren en las reuniones a puerta cerrada de un Gobierno o un partido político (encontronazos, rivalidades, pactos... etc.). Dentro de «informes escritos» se incluyen aquellas ocasiones en la que los periodistas publican una noticia adelantándose al comunicado oficial. 
f) Medios. La información ha sido obtenida por el periodista a través de la lectura de otros medios o de teletipos. Esta categoría no debe confundirse con la de «agencias/medios» en la subvariable Esfera. Por ejemplo, si el canciller alemán realiza unas declaraciones a Der Spiegel y la prensa española las toma prestadas, en la subvariable Esfera se consignará «presidente de Gobierno», y en la subvariable Estrategia, «Medios».

g) Archivo. Esta categoría contempla todas aquellas ocasiones en las que los datos o declaraciones se han obtenido documentándose a partir de lo ya publicado en el pasado.

h) Entrevista. Encuentro acordado entre periodista y fuente para la consecución de información que será reproducida en el diario bajo el formato pregunta-respuesta o mediante estilo indirecto.

i) Preguntas exclusivas. La información se consigue tras efectuar el periodista una llamada o ponerse en contacto con una fuente por iniciativa propia, o mediante una investigación efectuada a título individual por el corresponsal.

j) Observación. Se accede a la información mediante la presencia directa en el lugar de los hechos.

Diferenciar las estrategias no resultó fácil. Los periodistas no suelen precisar cómo se producen sus contactos con las fuentes y cuando lo hacen puede que exageren su iniciativa personal en la operación. Así, «documentos» y «ruedas de prensas» son fácilmente confundibles, ya que muchas veces el portavoz de una institución celebra un encuentro con los corresponsales precisamente para explicar un comunicado. Ante la ausencia total de pistas, se privilegia siempre la opción «rueda de prensa», que incorpora una presunción de mayor actividad por parte del profesional.

En las categorías acto público, archivo, documento, rueda de prensa y medios puede considerarse que quien toma la iniciativa de informar es la fuente (estrategia pasiva). En el caso de filtraciones, entrevistas, observación, pasillos y preguntas exclusivas, se estima que es el periodista quien lo hace (estrategia activa).

La distinción entre ambos grupos es importantísima y permite comprobar hasta qué punto la información que se obtiene a través de Internet es la que las fuentes quieren que se reciba o resulta del esfuerzo de los periodistas por dar a conocer esa otra realidad que «alguien en una parte del mundo trata de ocultar».

12. Presencia en Internet. Es una variable básica en este análisis que refleja si para cada noticia analizada existe un registro-fuente digital correspondiente, es decir, una nota de prensa, informe, archivo de audio o vídeo que fue difundido en la Red y que pudo actuar de manera efectiva como precursor.

Las categorías son un valor numérico $(1,2,3 \ldots$ etc.) compartido con su registro digital correspondiente, de modo que archivo digital y archivo en papel quedan vinculados por un número. Cuando la noticia carece de correspondiente digital, la categoría que se consigna es «0». 
Los registros digitales, para estar presentes en el análisis, deben corresponderse al menos con una noticia. No obstante, algunos se relacionan con varias, bien porque El Mundo y El País publican sobre el mismo tema o bien porque un medio «despieza» en varias noticias un mismo archivo digital. En este segundo caso, el archivo digital es duplicado en el análisis para poder estudiar por separado si contiene las claves informativas, políticas y ambientales de cada noticia que le corresponde.

\section{VALIDEZ DEL ESTUDIO Y PERSPECTIVAS}

Como ya se señaló al inicio, este artículo no pretende tanto exponer los resultados del estudio al que se viene haciendo referencia como relatar qué opciones metodológicas llevaron a sus conclusiones. Y ello porque al ser estos pasos, los referidos a la metodología, los que resultaron más costosos, pensamos que divulgarlos podría ayudar a investigadores que encuentren tesituras similares. Sea como fuere, y pensando especialmente en los lectores que se sientan perdidos con todas las variables descritas, puede volverse brevemente a los principales hallazgos obtenidos en la investigación original ${ }^{13}$.

El estudio confirma la hipótesis de partida («Internet se configura como un sistema de fuentes beneficioso para el periodista internacional, con capacidad para transformar sus rutinas de producción y de relación con los actores informantes»), pero lo hace con algunas salvedades. Son las siguientes:

- Más de un 37\% de las informaciones elaboradas por los corresponsales españoles en Bruselas podría haberse obtenido sin presencia física en el lugar de destino, gracias a los datos ofrecidos en Red. Internet, por tanto, sí proporciona al periodista un porcentaje lo suficientemente elevado de noticias como para quedar incluido entre sus puntos diarios de búsqueda de información.

- La Red facilita algunas de las tareas periodísticas más repetitivas; en concreto, en algunas ocasiones permite llevar a cabo virtualmente aquellas estrategias de contacto en las que son las fuentes las que toman la iniciativa de informar. Nos referimos a la celebración de ruedas de prensa, la difusión de comunicados, la puesta a disposición de la prensa de archivos históricos y la oferta pública de documentación. Todos estos acontecimientos o situaciones ya pueden vivirse en línea. Cuando una noticia se ha originado a partir de una estrategia pasiva, tiene una posibilidad de germinar en Internet del 47,7\%; el porcentaje es mucho más reducido cuando no hay voluntad explícita de comunicar por parte de las fuentes (ante un acontecimiento que perjudica su propia imagen, por ejemplo).

${ }^{13} \mathrm{El}$ lector que lo desee puede consultar tales conclusiones en toda su amplitud en diversos artículos ya publicados (Redondo, 2005; Redondo, 2007), 
- Por ahora la Red, y aquí está la principal decepción, no está corrigiendo posibles tendencias no deseables que se dan entre los profesionales, como el recurso excesivo a las declaraciones realizadas por fuentes de información de carácter privilegiado y elitista. Hasta un 92,8\% de los registros digitales útiles encontrados durante el estudio fueron hallados en salas de prensa digitales de alguna institución oficial; sólo el resto (y no en su totalidad) podría atribuirse a informantes alternativos o al llamado periodismo ciudadano. Además, el análisis de contenido mostró que la probabilidad más alta de que una noticia se halle en Internet $(54,96 \%)$ se da cuando su fuente principal es una gran institución, a menudo respaldada por un gabinete de comunicación con recursos económicos y bien engrasado.

Para quien haya leído hasta aquí manteniendo dudas sobre la fiabilidad del estudio, existe un argumento más a favor de su oportunidad. Tratándose de un análisis de contenido tan sui géneris, se decidió contrastarlo con la opinión de los propios corresponsales mediante la introducción en la investigación de una metodología que iba a ser exigida por los más críticos. Nos referimos a la encuesta, cuya desestimación como punto de partida en este caso - nunca como técnica general de estudio - responde a motivos que ya se explicaron a comienzo del artículo.

En noviembre de 2006 se hizo llegar a todos los corresponsales españoles destacados en Bruselas (58) un amplio cuestionario referido a su interacción con Internet. Aunque se trata de un conjunto reducido, tiene evidente interés como contrapeso a la información aquí facilitada y como grupo focal.

Los resultados no pueden divulgarse aquí en su totalidad por hallarse en proceso paralelo de publicación. No obstante, puede confirmarse sin ambages que ninguna de las conclusiones obtenidas mediante el análisis de contenido queda invalidada por las declaraciones de sus protagonistas. Las aportaciones de los corresponsales con respecto a las tres hipótesis presentadas aquí pueden resumirse como sigue:

- Internet proporciona suficientes datos de interés como para ser considerado un sistema de fuentes atractivo. Tanto es así, que los periodistas españoles de Bruselas navegan diariamente y suelen documentarse y contrastar la información en línea. No obstante, la consulta digital está lastrada por dos creencias: en Red, la autoría de las informaciones no siempre es clara, es fácil toparse con mentiras y rumores y hay mucho «aficionado».

- La Red facilita algunas de las tareas periodísticas más repetitivas. Así, según el 78,6\% de los periodistas que contestaron al formulario, el correo electrónico se utiliza cada vez más para contactar con las fuentes, y según el $57 \%$, se ha reducido la asistencia a actos públicos retransmitidos en línea. 
- Que los corresponsales naveguen por Internet no significa que estén ampliando su abanico de fuentes en beneficio de portavoces más alternativos. Creen que en el futuro podrán hacerlo y les gustaría, pero no es una realidad por el momento. Antes bien, los favoritos de los reporteros consultados giran en torno a SPD de instituciones oficiales internacionales, grandes medios de comunicación tradicionales o Gobiernos. Son, en definitiva, sitios privilegiados.

Las posibilidades cruzadas que ofrecen las variables descritas en este estudio nos parecen muy numerosas. Permitieron responder a las hipótesis planteadas inicialmente y creemos que permitirían servir a otras varias. Como sugerencia, se plantean aquí las siguientes preguntas:

- ¿Qué cantidad de noticias publicadas por la prensa pueden extraerse cada día de Internet sin necesidad de enviar a profesionales al lugar de los hechos?

- ¿Qué tipo de sitios web son los que proporcionan informaciones más útiles a los periodistas profesionales?, ¿las salas de prensa institucionales, la blogosfera, los foros?

- ¿Qué fuentes (privilegiadas o no privilegiadas, oficiales o no oficiales) influyen más en el discurso en Red?

- ¿Hasta qué punto resulta útil para los reporteros la información que las fuentes alternativas divulgan en Internet? ¿Cuál es su eficiencia digital? ¿Qué fallos se producen?

- ¿Qué mundos geográficos está privilegiando la Red? ¿hay un aumento de los países mencionados en línea con relación a los países en los que solían fijarse los medios de comunicación tradicionales?

Durante la ejecución del estudio que ha dado origen a estas preguntas, las dudas de procedimiento fueron numerosas, pero no insalvables. Y aunque alguna de las 20 variables que se han empleado pudiera ser contestada de manera específica, el funcionamiento, la marcha rítmica del conjunto, es real, algo que respalda la utilidad práctica de la propuesta de partida. La máquina se mueve.

Analizar Internet como fuente de información con fundamento científico todavía no es sencillo ni se conocen para ello caminos infalibles; pero no intentarlo sería un error.

\section{BIBLIOGRAFÍA}

Berelson, B. (1952): Content analysis in communication research, Nueva York, Free Press.

BERGER, A. A. (1991): Media research techniques, Newbury Park, SAGE. 
De Semir, V.; Ribas, C. y Revuelta, G. (1998): «Press releases of science journal articles and subsequent newspaper stories on the same topic», JAMA, vol. 280, n. ${ }^{\circ} 3$, julio, pp. 294-295.

Gans, H. J. (1979): Deciding what's news. A study of CBS Evening News, NBC Nightly News, Newsweek and Time, Nueva York, Pantheon Books.

GofFman, E. (1974): Frames analysis. An essay on the organization of experience, Nueva York, Harper and Row.

Golding, P. y Elliot, P. (1979): Making the News. London, Longman.

GRANT, W. (1989): Pressure groups politics and democracy in Britain, Londres, Philip Allan.

GunTER, B. (2002): «The quantitative research process», en Jensen, K. B. (ed.): A handbook of media and communication research. Qualitative and quantitative methodologies, Londres, Routledge.

HANITZSCH, T. (2006): «Network journalism studies. Towards a World Journalism Survey». Journalism Brazil Conference, noviembre de 2006. En línea [pdf: http://www.sbpjor.ufsc.br/pdf/palestras/thomas.pdf].

IYENGAR, S. y KINDER, D. R. (1987): News that matters: agenda setting and priming in a television age, Chicago, University of Chicago Press.

KuO-JEN, T., YeAn TASi y SCOTT, S. K. LiU. (1988): «Methodological emphases of international news studies», International Communication Bulletin, vol. 23, pp. 13-15.

LiPPMANN, W. (2003): La opinión pública, Madrid, Langre.

PIÑUEL, J. L. y GaITÁN, J. A. (1995): Metodología general. Conocimiento científico e investigación en la comunicación social, Madrid, Síntesis.

Pollock, J. C. (1981): The politics of crisis reporting. Learning to be a foreign correspondent, Nueva York, Praeger.

REDONDO, M. (2005a): «Internet como fuente de información en el periodismo internacional. Bruselas como caso de estudio», comunicación presentada al VI Congreso de Periodismo digital, Huesca, Asociación de la Prensa de Aragón.

REDONDO, M. (2005b): Internet como fuente de información en el periodismo internacional, Madrid, Universidad Complutense de Madrid.

REDONDO, M. (2007): «Pasividad y permisividad en Red: Internet y las nuevas actitudes de los periodistas», Comunicación y Sociedad, vol. XX, n. ${ }^{\circ}$ 3, julio 2007 (en imprenta).

Rivas Troitiño, J. M. (1992): Desinformación y terrorismo: Análisis de las conversaciones entre el Gobierno y ETA en Argel (enero-abril 1989) en tres diarios de Madrid, Madrid, Universidad Complutense de Madrid.

SMith, A. (1983): Goodbye Gutenberg. La revolución del periodismo electrónico, Barcelona, Gustavo Gili.

SREBERNY-MoHammady, A. (1995): «TV news flow and studies revisited», Electronic Journal of Communication, vol. 5, n. ${ }^{\text {os }} 2$ y 3, págs. 7-17.

Sreberny, A. y Stevenson, R. L. (cords., 1997): Cooperative Study of Foreign News and International News Flow [En línea: http://www.ibiblio.org/newsflow/index.html].

Sreberny-Mohammady, A., Nordenstreng, K., Stevenson, R. et al . (eds., 1985): Foreign news in the media. International reporting in 29 countries, París, UNESCO.

STEVENSON, R. L. y SHAW, D. L. (1985): Las noticias internacionales y el nuevo orden de la información mundial, Barcelona, Mitre.

TsanG, K. J., TsAI, Y. y LiU, S. K. (1988): «Methodological emphases of international news studies», International Communication Bulletin, vol. 23. 
Tuchman, G. (1978): La producción de la noticia, Barcelona, Gustavo Gili.

- (2002): «The production of news», en Jensen, K. B. (ed.): A handbook of media and communication research. Qualitative and quantitative methodologies, Londres, Routledge, 2002.

TúÑEz, M. (1999): Producir noticias. Cómo se fabrica la realidad periodística, Santiago de Compostela, Tórculo.

Wolf, M. (1987): La investigación en la comunicación de masas, Barcelona, Paidós. 


\title{
RESUMEN
}

Hasta el momento, la Red ha recibido más atenciones como medio de comunicación que como sistema de acceso a fuentes de información, quizá porque este último perfil es más escurridizo y presenta dificultades metodológicas. Sin embargo, es precisamente el comportamiento de Internet como fuente el que afecta a mayor número de periodistas, pues a la Red pueden acudir a extraer datos todos los profesionales, y no sólo los «ciberperiodistas» con el objetivo de publicar en ella como medio. El siguiente texto plantea la posibilidad de que una metodología comparativa centrada en un análisis de contenido dual (realizado simultáneamente sobre archivos digitales emitidos por las fuentes y noticias publicadas por los medios tradicionales) permita acercarse a ese campo desconocido. Se trata de un método que, partiendo del empleado por la UNESCO en los años 80, introduce conceptos novedosos y arriesgados como los de «clave informativa» y permite una nueva vía cuantitativa/cualitativa por la que acercarse a las rutinas digitales de los profesionales.

\section{PALABRAS CLAVE}

Internet, fuentes, rutinas, periodismo.

\begin{abstract}
Up to the moment, the Net has received academic attention mainly as a media and not a system of access to information sources. This is probably owed to the fact that the latter profile is more elusive and presents more methodological difficulties. Nevertheless, it is precisely Internet behaviour as «source» the one that affects a major number of journalists, since every professional (radio, tv, etc.) can extract information from the WWW, and not only the «cyberjournalists» who publish on the Net. The following text raises the possibility of studying Internet as a system of sources through a dual content analysis undertook simultaneously on the digital files published by the sources and on the news elaborated by the traditional media. The method — partly adapted from a huge research undergone by UNESCO in the 80s-introduces new and maverick concepts (such as the one of «informative key») and marks a new quantitative/qualitative route to approach digital journalistic routines.
\end{abstract}

\section{KEY WORDS}

Internet, sources, routines, journalism. 